\title{
A Survey on Unicast Routing Protocols for VANET
}

\author{
Imtisunep T Longchar, Rwngouti Chamframary, Dip Jyoti Deka, T Sivakumar
}

Dept. Of Computer Science, Pondicherry University

\begin{abstract}
VANET or Vehicular Ad-Hoc Network is an Ad-Hoc system where the vehicles acts like wireless nodes in order to communicate with each other on the road. Vehicles move at different speeds; thus creating a dynamic topology because the vehicles move out of the network; and new vehicles join the network.Because of the frequent changing topology of VANET it becomes difficult to route the packets effectively. In this survey paper, we study the unicast protocols used for routing in VANET. The main objective of VANET's is to basically create an Intelligent Transport System.
\end{abstract}

Keywords- VANET, Routing, Intelligent Transport System, Delay Tolerant, Delay Intolerant.

\section{INTRODUCTION}

VANET or Vehicular Ad-Hoc Network is an Ad-Hoc system where the vehicles acts like wireless nodes in order to communicate with each other on the road. Vehicles move at different speeds; thus creating a dynamic topology because the vehicles move out of the network and new vehicles joins the network. Because of the frequent changing topology of VANET it becomes difficult to route the packets effectively. In this survey paper, we study the unicast protocols used for routing in VANET. The main objective of VANET's is to basically create an Intelligent Transport System. The content of the paper is organised as: section II gives a detailed description of unicast protocols for routing in VANETs and its classification and section III is about conclusion and a tabulation showing the comparisons of the various unicast routing protocols.

\section{A. Applications of VANETs}

VANET's have a great deal of applications in our day to day life. Considering urban areas, the number of vehicles are growing exponentially everyday which triggers the traffic and road accidents to increase rapidly. With heavy traffic means we are wasting time which is a very important de-facto in this era.So, in this situation VANET's come into play. Through VANET, all the vehicles will be guided in an orderly manner, that is, every vehicles can communicate with each other so that the drivers are aware of the current situation of the traffic and can divert to a different route,resulting in minimizing the traffic and people needs less time to waste by being stuck in traffic.

In the case of safety applications, collision avoidance warning can notify the drivers of the vehicles of the possible collision. This means that, for instance, there is a turning and two vehicles are coming in either direction and these vehicles are not aware of that and can lead to serious accidents. But through VANET, both the vehicles will be able to communicate and becomes aware of the warning hence avoiding the possible accident. But as we know that every coin has two sides, sometimes accident does occur. Eventhough accidents may occur, but this does not limit the application of VANET. Incase of accidents, then the rescue vehicles can easily know the position of the accident by receiving the exact coordinates of the location and attend to the victims as soon as possible.

VANET's also provides comfort applications through internet connectivity that can help the passengers to listen, watch, download different types of media like movies, music, etc. online.

\section{Unicast Routing Protocols}

Unicast Routing Protocol in VANET aims at data transmission from one source to one destination by means of transmission through wirelessly multi-hopping or in a carrying-and-forwarding mannerism. In wirelessly multihopping transmission approach, the vehicles within the routing path transmits the data from the source vehicle to the destination vehicle at the earliest possible time.. In the carrying-and-forwarding approach,the vehicles which acts as source carries the packets as long as possible inorder to reduce the number of data packets.

Unicast Routing Protocol can be categorised into:-

(i) Delay Tolerant Routing Protocol

(ii) Delay Intolerant Routing Protocol.

Both these protocols are explained below.

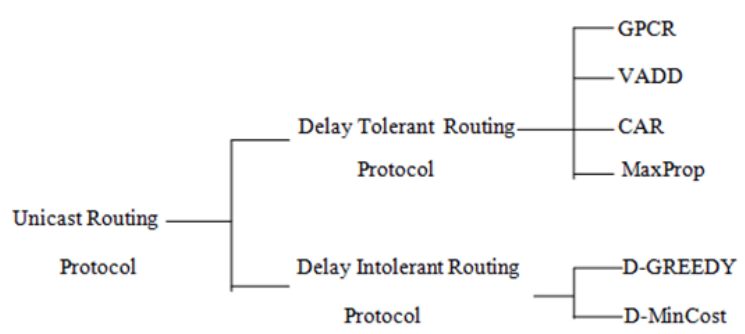

Fig 1: Classification of Unicast Routing Protocols for VANET

\section{A. Delay Tolerant Routing Protocol.}

The Delay Tolerant Protocol aims at transmitting the packets to the destination as early as possible by adapting the shortest-routing path with the primary interest of this protocol as the transmission delay time.

The different types of Delay Tolerant Protocols are:-

1) Greedy perimeter co-ordinator routing protocol (GPCR) :Greedy perimeter co-ordinator routing (GPCR) algorithm [3] is a revised form upon Geographic Source Routing Algorithm [4]. Unlike GPS, GPCR is independent of the external map of the street. According to James Bernsen and D.Manivannan [5] GPCR consist of two 
factors - a restrained greedy forwarding scheme and a reconstruction scheme for routing onward the road towards the target when the greedy strategy reaches a local optimum.

GPCR transmits data access across the street constantly. To curb the data transmission when an obstacle basically a turning or a skyscraper or intersection exist, GPCR has adopted a method in which it transmits the data to a node at the intersection called "co-ordinators" and then the "co-ordinators" makes the routing decisions.

To determine the coordinators, two approaches are known namely - Neighbor Table Approach and Correlation Coefficient Approach [3].

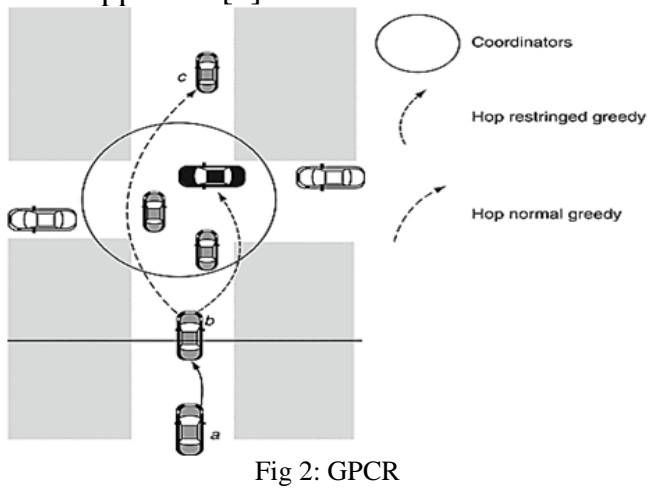

2) Vehicle assisted data delivery routing protocol (VADD) :The Vehicle assisted data delivery (VADD) algorithm [7] aims at enhancing routing even when the network is disconnected by employing the carry-andforward technique. Selection of a forwarding path with the minimum packet-delivery delay is considered to be the primary aim of VADD. So, the best path to forward the packets is selected by switching between the packet modes i.e. intersection, straightway and destination as shown in the figure below.

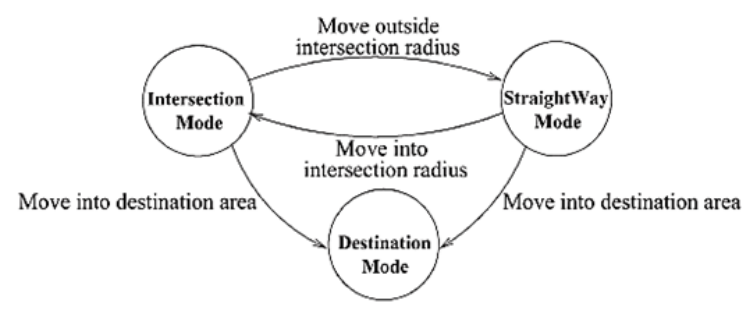

Fig 3: Transition modes of VADD
3) Connectivity Aware Routing protocol (CAR) :Connected aware routing protocol (CAR) [8] is a type of Position-based Vehicular protocol for routing. Frequent disconnection in the network is the most common and unavoidable problem in VANET and selection of the best path for forwarding the packets is one of the most important issues in VANET. So, as a counter part for these issues, CAR was developed. It calculates the possibility of every road section connectivity and then chooses the route with the maximum possibility of connection for forwarding the packets. CAR has the capability to manage a cache of routes which are succesful between the source and the target. Incase the destination vehicles changes its position, CAR can predict the positions of target vehicles by reconstructing the routes as those positions changes and deploys geographic marker messages.

4) MaxProp :MaxProp [9] is a type of delay tolerant protocol carried out in a real network called UMass DieselNet, where it is deployed on buses. It allows the buses to communicate amongst themselves and other wireless access point critical data such as performance information and location(via GPS) by utilising carry-andforward and packet prioritizing technique.

MaxProp operates in three stages namely:-

- Neighbor discovery stage: Each node will discover other like nodes within their range.

- Data transfer stage: Data is transferred among the nodes on the basis of maximum priority first scheme.

- Storage Management Scheme: Packets are deleted from the buffer by lowest priority first scheme.

Messages stored in the node buffer are prioritized based on the approximated delivery cost of the message to its target.

\section{B. Delay Intolerant Routing Protocol.}

Delay Intolerant Routing Protocol[9,10] is a carryingand-forwarding proposition where transmission of data is attempted from vehicles to fixed access points by maintaining low level of channel utilization while satisfying user-defined delay. Two types of Delay-Bounded Routing Protocols are:-

- D-Greedy Delay Intolerant Routing Protocols.

- D-MinCost Delay Intolerant Routing Protocols.

Table 1: Comparison Among Various Unicast Routing Protocols In Vanet

\begin{tabular}{|l|c|c|c|c|c|c|}
\hline \multicolumn{1}{|c|}{ Protocols } & GPCR & VADD & CAR & MaxProp & G-GREED & G-MinCost \\
\hline $\begin{array}{l}\text { Type of Routing } \\
\text { Protocol }\end{array}$ & $\begin{array}{c}\text { Based on } \\
\text { Geo }\end{array}$ & $\begin{array}{c}\text { Based on } \\
\text { Position }\end{array}$ & $\begin{array}{c}\text { Based on } \\
\text { Position }\end{array}$ & $\begin{array}{c}\text { Based on } \\
\text { Position }\end{array}$ & $\begin{array}{c}\text { Based on } \\
\text { Position }\end{array}$ & $\begin{array}{c}\text { Based on } \\
\text { Position }\end{array}$ \\
\hline Traffic Awareness & No & Yes & Yes & Yes & Go & No \\
\hline $\begin{array}{l}\text { Forwarding } \\
\text { Strategy }\end{array}$ & Greedy & Optimum & Greedy & Greedy & No & Yes \\
\hline $\begin{array}{l}\text { Map-based } \\
\text { Requirement }\end{array}$ & No & Yes & Yes & Integrated & Specialised & Specialised \\
\hline $\begin{array}{l}\text { Destination } \\
\text { Location Method }\end{array}$ & Specialised & Specialised & Integrated & Urban & Urban & Urban \\
\hline $\begin{array}{l}\text { Communication } \\
\text { environment }\end{array}$ & Urban & Urban & Urban & & & \\
\hline
\end{tabular}


D-Greedy and D-MinCost algorithms uses either data muling or multi-hop forwarding strategies [9] to minimize communication overhead after evaluation of the traffic information and bounded delay time.

1) D-Greedy Delay-Bounded Routing Protocols:DGreedy makes use of the local information about the traffic to the access point from the digital map and assigns time delay to the roads within the shortest paths. The packets must be delivered within the assigned time delay. Data muling strategy is used if packets can be transmited to the destination within the time delay. Otherwise multi-hop forwarding is used.

2) D-MinCost Delay Intolerant Routing Protocols:D-MinCost Delay Intolerant Routing Protocol uses global traffic information in order to minimize channel usage within the entrusted time delay. The cost and delay of every street is first computed from the global traffic information. The optimum path for routing with the least channel usage within the entrusted time to delay is selected using Delay Scaling Algorithm (DSA)[11].

\section{CONCLUSION}

In this paper, we have studied and surveyed on some of the unicast routing protocols for VANET which already exists. There are two types of forwarding strategies used by these protocols. One is "Greedy forwarding" and the other is "Optimum forwarding”. Position-based routing algorithm is found to perform better under high mobility.

Table 1 presents the comparison among various unicast routing protocols in VANET The prospective applications of Vehicular Ad-Hoc Network (VANETs) offers expansive opportunities, but still more efforts are required for the improvement and efficiency of VANETs so that it can be employed practically.

\section{REFERENCES}

[1] Vehicular communication ad hoc routing protocols: A survey, Baraa T. Sharef, Raed A. Alsaqour and Mahamod Ismail, Journal of Network and Computer Applications 40(2014) 363-396

[2] Routing in Vehicular Ad-Hoc Networks: A Survey, Fan Li and Yu Wang, IEEE Vehicular Technology Magazine,2007.

[3] C. Lochert, M. Mauve, H. Füÿler, H. Hartenstein, Geographic routing in city scenarios, ACM SIGMOBILE Mobile Computing and Communications Review 9 (1) (2005) 69_72.

[4] C. Lochert, H. Hartenstein, J. Tian, H. Füÿler, D. Hermann, M. Mauve, A routing strategy for vehicular ad hoc networks in city environments, in: Proceedings of the IEEE Intelligent Vehicles Symposium, 2003.

[5] Unicast routing protocols for vehicular ad hoc networks: A critical comparison and classification, James Bernsen, D. Manivannan, Pervasive and Mobile Computing 5 (2009).

[6] VADD: Vehicle-Assisted Data Delivery in Vehicular Ad Hoc Networks Jing Zhaoand GuohongCao,IEEE Transactions On Vehicular Technology, vol. 57, NO. 3, MAY 2008

[7] Connectivity Aware Routing in Vehicular Networks, Qing Yang and Alvin Lim and Agrawal ,2008 IEEE.

[8] J. Burgess, B. Gallagher, D. Jensen, B.N. Levine, MaxProp: Routing for vehicle-based disruption-tolerant networks, in: Proceedings of the 25th IEEE International Conference on Computer Communications, INFOCOM 2006, IEEE, 2006.

[9] Delay-bounded Routing in Vehicular Ad-hoc Networks, AntoniosSkordylis and NikiTrigoni, MobiHoc'08, May 26-30, 2008, Hong Kong SAR, China,ACM 978-1-60558-083-9/08/05

[10] Routing Protocols in Vehicular Ad Hoc Networks: A Survey and Future Perspectives*, Yun-Wei Lin1, Yuh-Shyan Chen2 And SingLing Lee1, September 30, 2009.

[11] A. Goel, K. G. Ramakrishnan, D. Kataria, and D. Logothetis, "Efficient computation of delay-sensitive routes from one source to all destinations," in Proceedings of IEEEConference on Computer Communications, 2001, pp. 854-858. 